\title{
How rational are indications for emergency caesarean section in a tertiary hospital in Tanzania?
}

\author{
MSELENGE H. MDEGELA ${ }^{1}$, PROJESTINE S. MUGANYIZI ${ }^{*}$, ANDREA B. PEMBE ${ }^{2}$, DAUDI O. SIMBA ${ }^{3}$ and \\ JOS VAN ROOSMALEN 4,5 \\ ${ }^{1}$ Liverpool School of Tropical Medicine, Liverpool, United Kingdom \\ 2Department of Obstetrics and Gynaecology, School of Medicine, Muhimbili University of Health and Allied Sciences, \\ Dar es Salaam, Tanzania \\ ${ }^{3}$ Department of Community Health, School of Public Health and Social Sciences, Muhimbili University of Health and \\ Allied Sciences, Dar es Salaam, Tanzania \\ ${ }^{4}$ Department of Obstetrics, Leiden University Medical Centre, The Netherlands \\ ${ }^{5}$ Section of Health Care and Culture, Free University Amsterdam, The Netherlands
}

\begin{abstract}
The rate of caesarean section (CS) at Muhimbili National Hospital (MNH) in Tanzania has been on progressive increase for past three decades. Concerns have been raised if this increase is justified by rational decisions but no study so far has investigated this problem. The aim of the study was to find out whether decisions made for CS comply with a set of locally made standards, with an assumption that if the standards are met, then the increase in CS rate seen at MNH is genuine. The five most common indications for CS were identified from the obstetric electronic data base. Most common indications included obstructed labour, cephalopelvic disproportion (CPD), failure to progress, repeat CS and foetal distress. Criteria for the best practice for each indication were developed based on the National guidelines and local expert consensus. Information extracted from the case notes, antenatal cards and partographs were compared to the standard audit criteria and the decision judged as standard or substandard. Three hundred forty five women had a decision made for emergency CS. Repeat CS was the most frequent indication $(30.2 \%)$, followed by obstructed labour (14.4\%) and foetal distress (13.6\%). Audit of 324 women's files showed that 30\% of women had substandard decisions for CS mostly in the foetal distress group (59.1\%) and least in the repeat CS group (9.1\%). Among the 324 mothers with decision for emergency CS, 279 $(86.1 \%)$ delivered by CS as decided and $45(13.9 \%)$ delivered vaginally before CS could be performed. Women who delivered vaginally after decision for CS and the nulliparous women had significantly more substandard decisions compared to those delivered by CS and parous women respectively. In conclusion, a substantial proportion of decisions for emergency CS made in the hospital is substandard and may contain women in whom surgical intervention could be avoided. This calls for a need to improve quality of assessment and decision before performing CS.
\end{abstract}

Keywords: decision, criterion-based audit, obstetric care, caesarean section, delivery, Tanzania

\section{Introduction}

The rates of caesarean section (CS) have progressively increased in high income (Bailit et al., 2004) as well as middle and low income countries (Gibbons et al., 2012; Maaloe et al, 2012a). In sub Saharan Africa, and other low income countries, the overall CS rates lag behind those in high income countries despite the high maternal and perinatal mortality rates in the former countries (Althabe et al., 2006; Gibbons et al., 2012; Maaloe et al., 2012a). In low income countries, characteristically the lowest CS rates are found in rural unlike in urban areas where hospital based studies indicate CS rates that are comparable with those in high income countries (Buekens et al., 2003; Gibbons et al., 2012; Maaloe et al., 2012a). The underlying causes of global increase in CS rates are multiple and could differ between countries, regions and hospitals within a country.

\footnotetext{
* Correspondence: Projestine S. Muganyizi; E-mail: promuga@yahoo.com
} 
These include emergency of CS on request, perceived safety of CS, socio-economic reasons, demographic changes, private practice, and others ( Bailit et al., 2004; Althabe et al., 2006; Belizan, Cafferata et al. 2006; MacDorman et al., 2008; Wylie et al., 2008; Zupancic 2008; Kringeland et al., 2009; Muganyizi et al., 2009; Scarella et al., 2011; Gibbons et al., 2012;Maaloe et al., 2012a; Maaloe et al., 2012b; Worjoloh et al., 2012). Similarly, strategies to reverse these rates cannot be universal. Although the primary aim of CS is to reduce maternal and perinatal morbidities and mortality, unnecessary performance of CS can bring about increased poor maternal and perinatal outcomes and higher financial costs to the already constrained health systems in developing countries ( van Roosmalen et al., 1995; Schuitemaker et al., 1997; Althabe et al., 2006; Daltveit et al., 2008; MacDorman et al., 2008; Wylie et al., 2008; Zupancic 2008; Gibbons et al., 2012).

Caesarean section rate at Muhimbili National Hospital (MNH) in Tanzania had increased from $15.7 \%$ in 1999 to $31.6 \%$ in 2004 and more than two thirds are performed as emergencies (Muganyizi et al., 2009). The rise of CS rate at this hospital could be explained by multiple factors including substandard decisions for CS. Moreover, studies in two rural hospitals in Tanzania have recently revealed suboptimal management of prolonged labour with low use of operative vaginal delivery methods (Maaloe et al., 2012b) and inappropriate indications for CS (Maaloe et al., 2012a). Studies elsewhere indicate that the use of objective criteria for to reach CS delivery decisions can reduce CS rates without compromising maternal and foetal wellbeing (Schuitemaker et al., 1997; Scarella et al., 2011). The aim of the current study was to find out whether decisions made for emergency CS deliveries comply with the local standards, with an assumption that if the standards are met, then the increase in CS rate seen at $\mathrm{MNH}$ is genuine.

\section{Materials and methods}

\section{Study setting}

Muhimbili National Hospital is the largest tertiary and University teaching hospital in Tanzania, located in Dar-es-Salaam city. Majority of deliveries come directly from home when the feel labour pains and only a third is referred from city municipal hospitals or other health facilities in the city and the nearby regions. Women who are admitted in the labour ward are attended by the admitting nurse who records patient's particulars and vital signs and initiates a partograph.

Everyday there is a team of doctors on call comprising of interns, resident, registrar and specialists. The interns, residents and registrar doctors are present in the labour ward at any time and commonly they see all admitted women and perform physical examinations including vaginal examination. The residents and registrars decide on management of the patient including CS and perform most of the CS. For the cases the resident/registrar finds difficult to manage, specialists are called to participate in the management. Once a decision to perform a CS is made, the patient is prepared and taken to theatre. On average there are thirty deliveries in a day among which approximately 30\% are decided for CS. All deliveries taking place at $\mathrm{MNH}$ are recorded into the delivery books and then the information is transferred into the electronic database. The information recorded includes socio-demographic characteristics, risk factors, complications before, during and after labour and HIV sero status.

\section{The audit criteria}

Five most common indications for emergency caesarean section for the year 2005 were identified from the $\mathrm{MNH}$ obstetric electronic database. These indications included obstructed labour, cephalopelvic disproportion (CPD), failure to progress, repeat CS and foetal distress. Diagnostic 
criteria were developed for each of these indications through review of the various National guidelines and tools (WHO 2003, MoHSW 2005a, MoHSW 2005b and MOHSW 2008) and the WHO (2003) integrated management of pregnancy and childbirth. The developed criteria were discussed among doctors and midwives in the department and an expert consensus reached. Each of the five major indications for SC had its own criteria for diagnosis and a composite of criteria that constitute a standard decision for CS. These audit criteria were piloted and necessary amendments made. For obstructed labour there were major and minor criteria and standard decision was made if two major criteria or one major and at least one minor criterion were present. For the diagnosis of repeat CS, it was subcategorized as repeat CS due to failed trial of labour or failure to progress without trial of labour. All decisions for emergency CS were judged substandard if the criteria for standard decision were not met.

\section{Sample size and data collection}

A sample size of 340 emergency Caesarean sections was calculated to adequately estimate the prevalence of suboptimal decisions in emergency setting, assuming a 32\% rate of such decisions, with a $95 \%$ confidence level and design effect of 1.0. This sample size would be obtained in six weeks at MNH. All mothers with a decision for CS made during the six weeks' period had their data extracted from files, antenatal cards, partographs and post operative notes. Data collection commenced January $26^{\text {th }}$ and ended March $7^{\text {th }} 2006$. The cases were then assigned to each of the five diagnoses or any other and decided if CS decision was standard or substandard based on set criteria. Once the first author did that, his decision was then discussed with the second and third author and the consensus was entered in the computer for analysis. In case a single case had more than one indication for CS the one which made the urgency for the operation was considered.

\section{Data analysis}

Data were entered into computer and cleaned using EPI Info version 6 programme. Analysis was performed by calculating proportions and frequencies. Chi-square test was used to compare proportions between women with standard and substandard decisions for caesarean section and socio-demographic characteristics. A p-value of less than 0.05 was taken as statistically significant.

\section{Ethical considerations}

Ethical clearance was granted by the Research and Publications committee of Muhimbili University College of Health Sciences and the permission to conduct the study was granted by $\mathrm{MNH}$ authorities. Anonymity was maintained during the review process to maintain confidentiality, however, the women were not asked for consent for the possibility of future use of the data for audit or research purposes.

\section{Results}

A total of 1,276 deliveries were conducted during the study period. Decision for CS was made in 423 (33.2\%) women, among which 345 (81.6\%) were decisions for emergency CS. Of the 345 women with decisions for emergency CS, 298 (86.4\%) had CS done and 47 (13.6\%) delivered vaginally before CS could be performed. Nineteen case notes of women delivered by emergency CS and two who delivered vaginally could not be found, thus remaining with 324 with a decision for emergency caesarean section for analysis. 
Table 1, shows that half of the women were between 20 and 29 years of age and one third were nulliparous women. Majority (87.3\%) of the women were married. Almost a quarter were referred to the hospital for delivery. Among the five indications, repeat CS was the most frequent indication consisting of one third of all indications, followed by obstructed labour $(14.8 \%)$ and foetal distress $(13.6 \%)$.

Table 1: Characteristics of women and their indications for emergency Caesarean section $(\mathbf{N}=324)$

\begin{tabular}{llll}
\hline Variable & Response & Number & Percent \\
\hline Age group (yrs) & $<20$ & 39 & 12.1 \\
& $20-24$ & 75 & 23.1 \\
& $25-29$ & 104 & 32.1 \\
& $30-34$ & 69 & 21.3 \\
Parity & $>34$ & 37 & 11.4 \\
Marital status & Nulliparous & 109 & 33.4 \\
& Parous & 215 & 66.6 \\
Admission status & Single & 30 & 9.3 \\
& Married & 283 & 87.3 \\
Indication for CS & Cohabiting & 11 & 3.4 \\
& Referred & 71 & 21.9 \\
& Not referred & 253 & 78.1 \\
& Repeat CS & 98 & 30.2 \\
& Obstructed labour & 48 & 14.8 \\
& Foetal distress & 44 & 13.6 \\
& Failure to progress & 40 & 12.3 \\
& CPD & 19 & 5.9 \\
& Others & 75 & 23.1 \\
\hline
\end{tabular}

Out of 324 women, 249 (76.8\%) had any of the five common indications for CS diagnosed and were audited against the set criteria. Overall $30.1 \%$ of all decisions for emergency CS were judged substandard. The percentage with substandard decision was highest $(60 \%)$ when foetal distress was the indication for emergency CS and lowest (10\%) in repeat caesarean section (Figure 1).

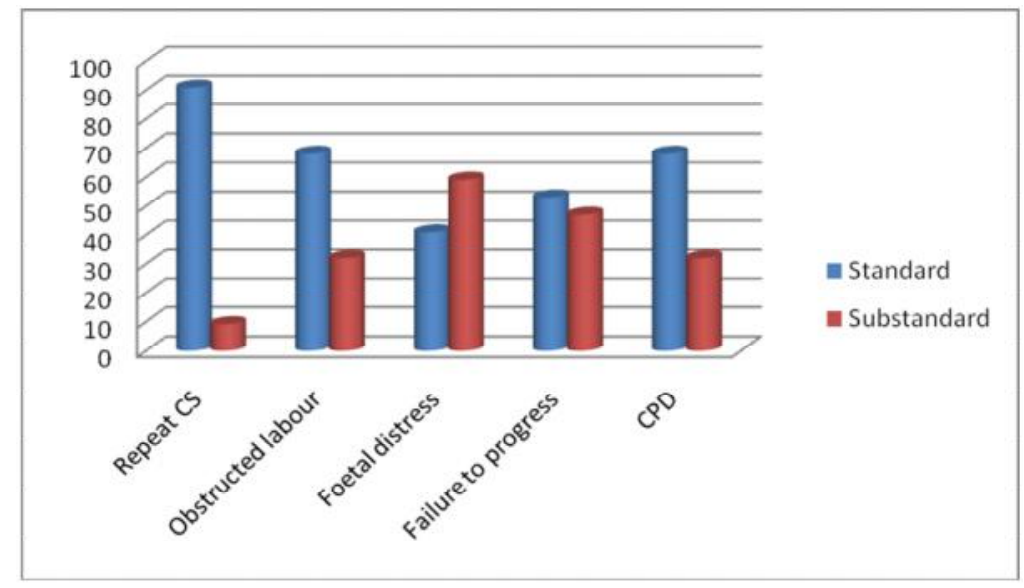

Figure 1: Percentage of indications for emergency CS meeting standard/substandard criteria $(\mathrm{N}=249)$

Fourteen percent of women with a decision for emergency CS delivered vaginally. This occurred most frequently in women with an indication of failure to progress $(26 \%)$, and least frequently in 
repeat CS (6\%). Even among those with an indication of obstructed labour almost 20\% delivered vaginally (Figure 2).

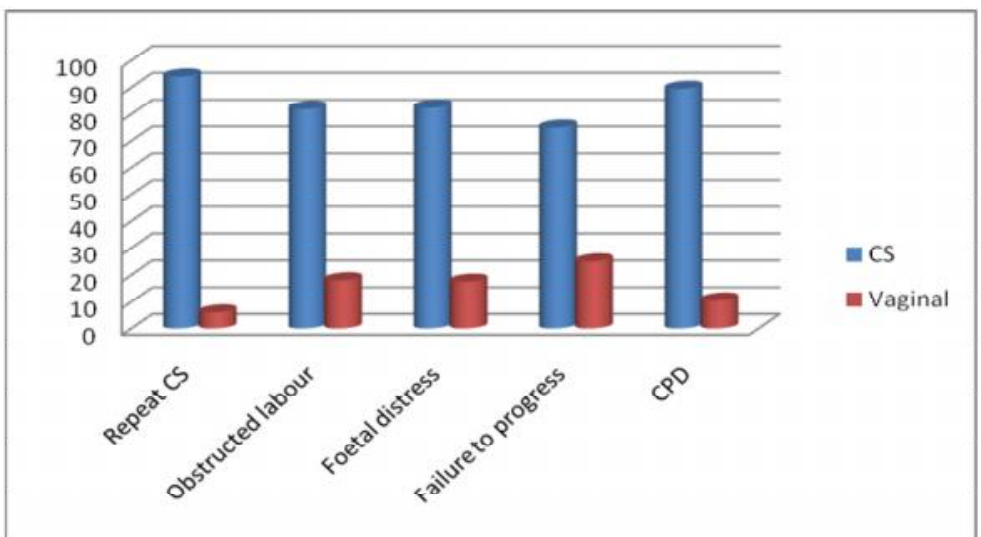

Figure 2: Ultimate mode of delivery among women with a decision for emergency CS by type of indication

Women who delivered vaginally after decision for emergency CS and the nulliparous had statistically significant more substandard decisions. There was no statistical difference among women referred and not referred to the hospital (Table 2).

Table 2: Stratified analysis of maternal characteristics according to standard and substandard decisions for emergency CS

\begin{tabular}{lllll}
\hline Characteristic & Total & \multicolumn{2}{l}{ Evaluation criteria } & p-value \\
\cline { 3 - 4 } & & $\begin{array}{l}\text { Standard } \\
(N=174) \\
\mathrm{n}(\%)\end{array}$ & $\begin{array}{l}\text { Substandard } \\
(N=75) \\
\mathrm{n}(\%)\end{array}$ & \\
\hline $\begin{array}{l}\text { Mode of delivery } \\
\text { CS }\end{array}$ & 214 & $162(75.7)$ & $52(24.3)$ & 0.001 \\
SVD & 35 & $12(34.3)$ & $23(65.7)$ & \\
Parity & & & & 0.003 \\
Nulliparous & 80 & $46(57.5)$ & $34(42.5)$ & \\
Parous & 169 & $128(75.7)$ & $41(24.3)$ & \\
Admission status & & & & \\
$\quad$ Referred & 52 & $40(76.9)$ & $12(23.1)$ & \\
$\quad$ Non referred & 197 & $134(68.0)$ & $63(32.0)$ & \\
& & & & \\
\hline
\end{tabular}

\section{Discussion}

We have observed that one third of all the deliveries conducted at MNH during the study period had decision for CS made and that a third of those with decisions for emergency CS had substandard decisions. This high rate of substandard decisions must have contributed significantly not only to the high burden of CS but also to substantial utilization of the meagre resources in this hospital (Zupancic, 2008). Substandard decisions for CS are not unique for MNH as it has been reported in many other countries irrespective of their levels of economic 
development. In USA, there has been a substantial increase in CS performed for no indicated risks and for the very low risk groups probably due to changes in obstetric practice (Bailit et al., 2004; MacDorman et al., 2008). In Chile, unnecessary CS were reported to contribute to at least $5 \%$ of all CS deliveries that could have been prevented without negative impact on the rate of fetal asphyxia (Scarella et al., 2011). A recent study in rural hospitals in Tanzania has reported similarly high percentage $(26 \%)$ of substandard decisions for CS (Maaloe et al., 2012a,b) indicating that though the problem seems to be universal, the burden of substandard CS deliveries in Tanzania could rather be on the highest side.

At $\mathrm{MNH}$ the majority of CS deliveries during the period of study were by emergency CS indicating that some women who could have been delivered electively came as emergencies. A different scenario exists in high income and Latin American countries where majority of the CS are elective operations (Belizan et al., 1999; Bailit et al., 2004; Belizan et al., 2006; Gibbons et al., 2012). Though it is difficult to confirm what proportion of emergency CS should have been delivered electively it can be stipulated that such women could have contributed significantly to the observed high rate of substandard decisions.

The current CS rate at MNH could probably be lowered safely if regular auditing and feedback could be implemented. The majority if not all of the $30 \%$ of substandard decisions would have been prevented if appropriate audit measures were in place since such measures have been effective in reducing substandard CS decisions elsewhere ( Scarella et al., 2011). Moreover, in the current study, the nulliparous and those who delivered vaginally after decision for CS were found to have more substandard indications, thus proving evidence that a substantial proportion of CS deliveries could be safely abated without jeopardy of foetal wellbeing. Having one-third of all emergency CS done at MNH being primary (first caesarean section), and having significantly more substandard decisions in this group could partly explain the increasing trend in CS rate. Wilkinson et al. (1998) reported that half of the CS deliveries in Scotland were done in nulliparous and more than half were emergencies in labour. This finding underscores the importance of preventing unnecessary primary CS as a cornerstone in reversing the present and future rates of CS (Myers et al., 1988; van Roosmalen et al., 1995; Belizan et al., 1999; Bailit et al., 2004; Belizan et al., 2006; Wylie et al., 2008; Scarella et al., 2011; Gibbons et al., 2012).

For the individual diagnoses, it was particularly of interest to find that among those who delivered vaginally many were diagnosed to have failure to progress than with previous scar or foetal distress. This implies that more women with this diagnosis could deliver vaginally, provided careful and appropriate intrapartum monitoring is done. This would have reduced the risk of maternal exposure to surgery; an important consideration if reduction of primary caesarean section is to be achieved. There is much overlap between obstructed labour, failure to progress and CPD. Together they have been grouped as labour dystocia by some authors (van Roosmalen et al.,1995) which implicitly makes labour dystocia the most frequent indication, followed by repeat CS and foetal distress.

Women for repeat CS included those with one scar who are potential candidates for vaginal birth. About $6 \%$ of women with at least one scar delivered vaginally after a decision for emergency CS were made. This finding gives a clue that more women with one previous scar could benefit if trial of labour was attempted (Paterson et al., 1991). More studies are needed in this area to ascertain local factors leading to a successful vaginal birth after caesarean section. Health workers should decide in advance the mode of delivery of women with a previous CS scar. Those who should not have a trial of labour should be counselled and booked for elective CS. Women who can have a trial of labour should be encouraged to report to hospital as soon as 
labour starts. This may help to reduce the rate of CS and number of emergency CS since emergencies are associated with more adverse outcomes than electives(Paterson et al., 1991).

Of all the five major diagnoses for emergency CS, that of foetal distress was more substandard than standard. The diagnosis of foetal distress needs more strict criteria and search for the cause of distress (WHO, 2003). It is often observed that the diagnosis of feta distress is made based only on modest meconium stained liquor without changes in foetal heart rate patterns which is a reversible phenomenon in labour(WHO, 2003; Daltveit et al., 2008). More objective methods such as tococardiography and foetal blood sampling are at the moment not widely practiced in Tanzania. In a more recent study in rural hospitals in Tanzania, foetal distress emerged the most misdiagnosed indication in wholly $84 \%$ indicating that this problem could be National wide (Maaloe et al., 2012a).

This study had some limitations. MNH is a tertiary and teaching hospital with different cadres taking care of the patients in the labour ward. We did not attempt to separate cases according to the cadres involved in making final decision on emergency CS. Separating the cases could have given more light to the areas to intervene among doctors deciding on CS. Furthermore being a tertiary hospital and urban setting, our results do not represent the real situation in the rural situated hospitals and the general population. Nevertheless, these results can be used to gauge the situation in lower level public hospitals especially in the urban. In conclusion, a substantial proportion of decisions for emergency CS made at MNH are substandard. This has contributed to the currently high CS rate in the hospital which calls for a need to improve quality of assessment and decision before performing CS.

\section{Acknowledgements}

We wish to acknowledge all specialists, resident and intern doctors, nurses, midwives and other staff MNH and MUHAS for their valuable contribution during development of the criteria and data collection. This study received financial the support from the Health Research Users' Trust Fund of the National Institute of Medical research.

\section{References}

Althabe, F., Sosa, C., Belizan, J.M., Gibbons, L., Jacquerioz, F. \& Bergel, E. (2006) Cesarean section rates and maternal and neonatal mortality in low-, medium-, and high-income countries: an ecological study. Birth 33, 270-277.

Bailit, J.L., Love, T.E. \& Mercer, B. (2004) Rising cesarean rates: are patients sicker? American Journal of Obstetrics \& Gynecology 191, 800-803.

Belizan, J.M., Althabe, F., Barros, F.C. \& Alexander, S. (1999). Rates and implications of caesarean sections in Latin America: ecological study. British Medical Journal 319, 1397-1400.

Belizan, J.M., Cafferata, M.L., Athabe, F. \& Buekens, P. (2006) Risks of patient choice cesarean. Birth 33, 167-169.

Daltveit, A.K., Tollanes, M.C., Pihlstrom, H. \& Irgens, L.M. (2008) Cesarean delivery and subsequent pregnancies.Journal of Obstetrics \& Gynecology 111, 1327-1334.

Gibbons, L., Belizan, J.M., Lauer, J.A., Betran, A.P., Merialdi, M. \& Althabe, F. (2012) Inequities in the use of cesarean section deliveries in the world. American Journal of Obstetrics $\mathcal{E}$ Gynecology 206, 331 e331-319.

Kringeland, T.A., Daltveit, A.K. \& Moller, A. (2009) What characterizes women in Norway who wish to have a caesarean section? Scandinavian Journal of Public Health 37, 364-371. 
Maaloe, N., Sorensen, B.L., Onesmo, R., Secher, N.J. \& Bygbjerg, I.C. (2012a) Prolonged labour as an indication for emergency caesarean section: a quality assurance analysis by criterionbased audit at two Tanzania rural hospitals. British Journal of Obstetrics \& Gynecology 119, 605-613.

Maaloe, N., Bygbjerg, I.C., Onesmo, R., Secher, N.J. \& Sorensen, B.L. (2012b) Disclosing doubtful indications for emergency caesarean section in rural hospitals in Tanzania: a retrospective criterion- based audit. Acta Obstetricia et Gynecologica Scandinavica 91, 1069-1076.

MacDorman, M.F., Menacker, F. \& Declercq, E. (2008) Cesarean birth in the United States: epidemiology, trends, and outcomes. Journal of Clinics in Perinatology 35, 293-307.

MoHSW (2005a) Antenatal care card (RCH-4) Dar es Salaam, Ministry of Health and Social Welfare, United Republic of Tanzania.

MoHSW (2005b) Advanced life saving skills curricula. Dar es Salaam, Ministry of Health and Social Welfare, United Republic of Tanzania.

MoHSW (2008) Emergency obstetric care job aid. Dar es Salaam, Ministry of Health and Social welfare, The United Republic of Tanzania.

Muganyizi, P.S. \& Kidanto, H.L. (2009) Impact of change in maternal age composition on the incidence of Caesarean section and low birth weight: analysis of delivery records at a tertiary hospital in Tanzania, 1999-2005. BMC Pregnancy Childbirth 9, 30.

Myers, S.A. \& Gleicher, N. (1988) A successful program to lower cesarean-section rates. New England Journal of Medicine 319, 1511-1516.

Paterson, C.M. \& Saunders, N.J. (1991) Mode of delivery after one caesarean section: audit of current practice in a health region. British Medical Journal 303, 818-821.

Scarella, A., Chamy, V., Sepulveda, M. \& Belizan, J.M. (2011) Medical audit using the Ten Group Classification System and its impact on the cesarean section rate. European Journal of Obstetrics \& Gynecology and Reproductive Biology 154, 136-140.

Schuitemaker, N., van Roosmalen, J., Dekker, G., van Dongen, P., van Geijn, H. \& Gravenhorst, J.B. (1997) Maternal mortality after cesarean section in The Netherlands. Acta Obstetricia et Gynecologica Scandinavica 76, 332-334.

van Roosmalen, J. \& van der Does, C.D. (1995) Caesarean birth rates worldwide. A search for determinants. Tropical and Geographical Medicine 47, 19-22.

WHO, United Nations Population Fund, UNICEF, The World Bank (2003) Managing complications in pregnancy and childbirth: A guide for midwives and doctors. World Health Organization, Geneva

Wilkinson, C., Mcllwaine, G., Boulton-Jones, C. \& Cole, S. (1998) Is a rising caesarean section rate inevitable? British Journal of Obstetrics and Gynaecology 105, 45-52.

Worjoloh, A., Manongi, R., Oneko, O., Hoyo, C., Kjersti Daltveit, A. \& Westreich, D (2012) Trends in cesarean section rates at a large East African referral hospital from 2005-2010. Open Journal of Obstetrics and Gynecology 2, 255-261.

Wylie, B. J. \& Mirza, F.G. (2008) Cesarean delivery in the developing world. Journal of Clinics in Perinatology 35, 571-582.

Zupancic, J. A. (2008). The economics of elective cesarean section. Journal of Clinics in Perinatology $35,591-599$. 